\title{
The Thirty Meter Telescope site testing robotic computer system
}

\section{Reed L. Riddle, Matthias Schöck, Warren Skidmore}

Reed L. Riddle, Matthias Schöck, Warren Skidmore, "The Thirty Meter Telescope site testing robotic computer system," Proc. SPIE 6267, Groundbased and Airborne Telescopes, 62671Q (23 June 2006); doi: 10.1117/12.672658

SPIE Event: SPIE Astronomical Telescopes + Instrumentation, 2006, Orlando, Florida, United States 


\title{
The Thirty Meter Telescope Site Testing Robotic Computer System
}

\author{
Reed L. Riddle, Matthias Schöck, and Warren Skidmore \\ Thirty Meter Telescope Project, 2632 E. Washington Blvd., Pasadena, CA, 91107 USA
}

\begin{abstract}
The Thirty Meter Telescope (TMT) project is currently testing six remote sites as candidates for the final location of the telescope. Each site has several instruments, including seeing monitors, weather stations, and turbulence profile measuring systems, each of which is computer controlled. As the sites are remote (usually hours from the nearest town), they requires a system that can control the operations of all the varied subsystems, keep the systems safe from damage and recover from errors during operation. The robotic system must also be robust enough to operate without human intervention and when internet connections are lost. It is also critical that a data archiving system diligently records all data as gathered. This paper is a discussion of the TMT site testing robotic computer system as implemented.
\end{abstract}

Keywords: Telescopes, robotic; site testing, telescope control software

\section{INTRODUCTION}

The Thirty Meter Telescope (TMT) project is currently testing six candidate sites for location of the TMT. With a project of this scope, putting it on the best available site is critical to maximizing the scientific benefit from the observatory. It was decided early on that the TMT site testing program would build on past programs by installing robotic telescopes that would remain on the sites continuously for a period of at least two years, gathering a uniform data set to use in selecting the observatory site, the design of the telescope, instruments and enclosure. To compile these measurements, an extensive suite of instrumentation has been brought together at each site to measure weather, atmospheric turbulence and other parameters that affect the suitability of a site for an astronomical observatory.

One of the challenges in instrumentation is creating software to control the instrument; many times software is the critical factor in how well (or poorly) instrumentation functions. In the case of the TMT site testing program, there are nine instruments, each with their own software (created by the site testing team, the manufacturer, or both), which all software must function reliably in order to gather data. Additional software is required to control the operations of site itself; with six remote sites, direct human interaction with the hardware is limited. A robust computer system that can control the instruments, keep the instrumentation safe from weather and recover from errors is critical to a successful remote operating station.

The TMT site testing computer system, as a whole, is comprised of 45 computers, with seven different operating systems installed, and 12 terabytes of disk space. The software controlling this is both manufacturer software and internally developed software, coded in twelve separate programming or scripting languages. The computer system is designed to accomplish the observations automatically without human intervention. The site testing program depends on the computer and instruments systems all working together to give us a complete picture of the conditions on the site, night after night.

\section{A GENERAL DESCRIPTION OF THE SITE TESTING INSTRUMENTS}

This section is a short summary to familiarize the reader with the equipment installed at the sites and the complexity of the system. A complete description of the instruments and subsystems used as part of the TMT site testing project will be included in a later publication after the completion of the site testing program.

Further author information: (Send correspondence to R. Riddle)

R. Riddle: E-mail: riddle@tmt.org, Telephone: 16263958956

M. Schöck: E-mail: mschoeck@tmt.org

W. Skidmore: E-mail: was@tmt.org

Ground-based and Airborne Telescopes, edited by Larry M. Stepp, Proc. of SPIE

Vol. 6267, 62671Q, (2006) · 0277-786X/06/\$15 - doi: 10.1117/12.672658

Proc. of SPIE Vol. 6267 62671Q-1 


\subsection{Robotic Telescope System}

The core of the site testing system is a $35 \mathrm{~cm}$ telescope produced by Teleskoptechnik Halfmann in Germany. This telescope was selected due to its robust nature; it is sturdier than the commercially available amateur telescopes usually used in site testing, and well suited to operations on a remote mountaintop.

The telescope comes with a fold away dome that opens fully to allow unobstructed airflow through the system (except through the bottom which is sealed with a tarpaulin). The entire telescope is open to the atmosphere during operations. An electronics cabinet has all of the equipment necessary to run the telescope system automatically, and a computer interface is provided for remote control and command, as described in Sec. 3.2.10. The telescope is mounted at the top of a $6.5 \mathrm{~m}$ tower.

\subsection{MASS/DIMM}

The Multi-Aperture Scintillation Sensor (MASS) is used to measure the atmospheric turbulence profile, as well as the isoplanatic angle. ${ }^{1}$ The MASS consists of a "segmentator" mirror consisting of four concentric sub-apertures in the pupil plane (one circular aperture and three annuli); each sub-aperture directs the star light to one of four photo multiplier tubes (PMT). Scintillation indices in each aperture, and between apertures, are used with specially designed weighting functions to calculate the atmospheric turbulence profile between about $0.5 \mathrm{~km}$ and $16 \mathrm{~km}$.

The Differential Image Motion Monitor (DIMM) measures the line of sight seeing and photometric conditions using a CCD image of the motion of a split stellar image. ${ }^{2}$ Two mirrors direct different portions of the telescope aperture to a Santa Barbara Instrument Group (SBIG) ST-7 CCD. ${ }^{3}$ The CCD operates in one of two modes: pointing, which uses CCD frames of the two stellar images to center the telescope; and DIMM data, which takes data frames of thirty second duration ever two minutes. See Sec. 3.2.5 for a description of the software that controls the DIMM.

Both instruments are mounted within one instrument body, which is attached to the telescope at the Cassegrain focus position. The MASS/DIMM instruments are made by the Cerro Tololo Inter-American Observatory (CTIO) instrument group in La Serena, Chile and the Sternberg Astronomical Institute in Moscow, Russia.

\subsection{Sonic Anemometer}

The sonic anemometer measures the three dimensional wind velocity and temperature at a frequency of $60 \mathrm{~Hz}$. Commercially available CSAT3 sonic anemometer units from Campbell Scientific are used in the TMT site testing project; they use small sound emitters and receivers, equally spaced over a sphere $\sim 10 \mathrm{~cm}$ in diameter, to measure atmospheric properties. ${ }^{4}$ One is mounted at the $7 \mathrm{~m}$ level outside the dome at each site. The wind and temperature measurements are used to calculate optical turbulence at the location of the sonic anemometer. The wind velocity and temperature time series measurements will be used to evaluate the performance of the TMT telescope and enclosure design.

\subsection{Dust Sensor}

Dust is the enemy of every telescope, as it coats optics and invades mechanical elements. A Met One Instruments, Inc. model GT-321 commercial dust sensor at each site monitors the particle environment. ${ }^{5}$ The dust sensor is mounted at the $7 \mathrm{~m}$ level, and uses a laser diode to measure the number of particles in the atmosphere of sizes $0.3 \mu \mathrm{m}, 0.5 \mu \mathrm{m}, 1.0 \mu \mathrm{m}, 2.0 \mu \mathrm{m}$ and $5.0 \mu \mathrm{m}$ every six minutes.

\subsection{SODAR}

Scintec SOund Detection And Ranging (SODAR) instruments, are set up on the ground at the candidate sites. ${ }^{6}$ These commercial devices are used at airports to detect wind velocities, turbulence and wind shear. There are two units: the large unit (XFAS), which can measure from $40 \mathrm{~m}$ to $800 \mathrm{~m}$; and the small unit (SFAS), which can measure from $15 \mathrm{~m}$ to $200 \mathrm{~m}$. The units measure the turbulence profile and three dimensional wind velocity in twenty minute intervals throughout the night; calibration measurements at noon are taken on occasion. 


\subsection{ASCA}

The All-Sky Camera (ASCA) is a replica of the unit mounted at CTIO, and is used in both the TMT and LSST site testing campaigns. ${ }^{7}$ ASCA takes an image of the entire sky once every five minutes through the night in four bands (b, r, y, z), and images every three hours using a Na filter. The images are used to determine the conditions of the night sky outside of the MASS/DIMM field of view. Currently, a determination of cloud fraction is made "by eye"; further software development is planned to measure automatically the cloud fraction, as well as photometric variations of the visible stars, in order to measure the transparency of the sky directly.

\subsection{Weather station}

A commercial weather station, produced by Monitor Sensors in Australia, is used to gather meteorological information at the site. ${ }^{8}$ The station measures wind speed and direction, humidity, air and ground temperatures, atmospheric pressure, radiation (solar and ground flux), and if there is dew, rain or snow forming at the site.

\subsection{IRMA}

The Infrared Radiometer for Millimetre Astronomy (IRMA), developed and built by a team from the University of Lethbridge in Canada, measures the water vapor content of the atmosphere (along the line of sight of the unit) every second. ${ }^{9}$ IRMA is also steerable, so that sky dips can be taken.

\subsection{Support Equipment}

As the sites are remote in most cases, the only viable option for electricity is solar power. The power systems at the remote sites consists of a solar power system built by Advanced Energy Group that outputs both 220V (for the Halfmann telescope) and $110 \mathrm{~V}$ (for the rest of the equipment), with a power output of $600 \mathrm{~W}$ continuous and $3 \mathrm{~kW}$ maximum. ${ }^{10}$

A continuous internet connection has been established at each site so the status of the system can be monitored at all times. On the remote sites, the network connection is through a microwave antenna system. On sites placed at an established observatory site, the network and power systems are supported through local infrastructure. Webcams for site monitoring (from Axis and TRENDnet), and network power switches (from Western Telematic Inc.) for remote instrument switching, are also installed at the sites and discussed in Sec. 3.2.2.

\section{SITE TESTING COMPUTER SYSTEMS AND SOFTWARE}

Table 1 summarizes the site testing instruments and equipment, and the connections required for communication. This suite of instruments and support equipment results in a complex computer configuration and operational system. Some forethought about the connections and computer requirements can simplify matters, so care was taken to consider the optimum configuration. There are many possible ways to solve the system puzzle; the following describes the system as implemented.

\subsection{Considerations for Connecting the Instrumentation}

The site testing instrument suite drives the design of the computer system that controls the operations of the site. Each piece of equipment has a specific connection required for communication and control, and requires some level of computer support to gather data. Ideally, everything would be connected through ethernet, allowing a single computer to orchestrate all of the equipment through the LAN. However, this is not possible with the current instrument suite. Different direct connections to computer systems for the non-ethernet based instruments and equipment are required. and at least two separate operating systems are necessary. These conditions drive the requirement that there be several separate computers in the system.

Reliability also is essential, as computers will fail at some point. Connecting all of the Linux based equipment together on one computer (for example) ensures that, at some point, the data flow would completely stop for the connected instruments. Spreading the connections out among separate computers allows at least some instruments to continue working when a computer fails. Multiple computers also allow multiple lines of access to the ethernet based equipment, where a single computer system would die completely with a computer failure. 
Table 1. A summary of the equipment comprising the site testing system and the connections required.

\begin{tabular}{|c|c|c|c|}
\hline Name & Manufacturer & Model & Connection \\
\hline Robotic Telescope & $\begin{array}{l}\text { Halfmann } \\
\text { Teleskoptechnik, } \\
\text { Germany }\end{array}$ & DIMM 1-6 & Onboard computer system, ethernet connection \\
\hline DIMM CCD & SBIG & $\mathrm{ST}-7$ & USB connection to Windows computer \\
\hline MASS & CTIO/Sternberg & None & Parallel (RS-485) connection to Linux computer \\
\hline Sonic Anemometer & $\begin{array}{l}\text { Campbell Scien- } \\
\text { tific }\end{array}$ & CSAT3 & Serial (RS-232) connection to Linux computer \\
\hline Dust Sensor & $\begin{array}{l}\text { Met One Instru- } \\
\text { ments }\end{array}$ & GT-321 & Serial (RS-232) connection to Linux computer \\
\hline SODAR & Scintec & $\begin{array}{l}\text { XFAS, } \\
\text { SFAS }\end{array}$ & $\begin{array}{l}\text { Serial (RS-232) connection to Windows com- } \\
\text { puter }\end{array}$ \\
\hline ASCA & CTIO & None & Onboard computer system, ethernet connection \\
\hline Weather Station & Monitor Sensors & None & Serial (RS-232) connection to Linux computer \\
\hline IRMA & $\begin{array}{l}\text { University of } \\
\text { Lethbridge }\end{array}$ & None & Onboard computer system, ethernet connection \\
\hline Webcam & $\begin{array}{l}\text { AXIS } \\
\text { TRENDnet }\end{array}$ & $\begin{array}{l}2100,2110 \\
\text { IP-TV100 }\end{array}$ & Ethernet connection \\
\hline Network Power Switch & $\begin{array}{l}\text { Western } \\
\text { Telematic Inc. }\end{array}$ & $\begin{array}{l}\text { NPS-115, } \\
\text { IPS-15CE }\end{array}$ & Ethernet connection \\
\hline
\end{tabular}

If the systems can operate reliably and recover from failures without requiring a visit to the site, then a higher quality data set for the program will be obtained.

Containing the various instruments and computers within sensible equipment subgroups also drives the system design. The SODAR software is processing power intensive, as is the DIMM reduction software, so running both on the same computer could affect operations. Installing separate Windows machines for each is an obvious step; this creates a self contained SODAR system (instrument and computer) and self contained DIMM system (instrument and computer). A similar grouping contains the sonic anemometer and dust sensor, as each requires a serial connection to a Linux systems and most computer systems come with two serial ports standard.

The MASS and weather station instruments have direct connections, but require additional consideration. The weather information must be directly monitored by the system that monitors the safety of operations for the telescope and site equipment. The MASS can be run by the system running the site operations, and its parallel port connection is easiest to handle directly. Connecting these instruments to the same computer that will supervise the operations of the entire system results in the most efficient system operation.

Examining the instruments and equipment in the site testing system, the requirement for a minimum of four computers (excluding instruments with internal computers) to run everything is obvious. Extra computers for redundancy, or to act as an overseer for the system, could be added to the system, and the instruments could be reorganized in a different fashion, but this logic is what was used to develop the system currently deployed. These considerations have also driven the software development, as software to run each individual instrument as well as to run the system as a whole is required.

\subsection{Pieces of the Puzzle}

The next step is to assemble the disparate set of equipment described above into a working suite of instrumentation that gathers accurate data. 


\subsubsection{Computer equipment}

Size, power requirements and heat control are considerations for a computer at a remote site. Each site has a weatherproof cabinet for all of the computer equipment; large computers would fill the cabinet quickly, allowing for little airflow or space for additional equipment, if necessary. Large computers also use more power, and the connection requirements made most of the benefits of a large computer irrelevant. Temperature is also a factor, as the site systems have no air conditioning to keep them cool. Stealth Computer builds a small computer enclosure that has all of the connections that we require, and that is designed to work safely at temperatures up to $+40^{\circ} \mathrm{C}^{11}$. The Stealths were selected for use as the main computers in the system, and they have performed reliably to this point.

\subsubsection{Support equipment: Power and Webcams}

The Halfmann telescope system power circuit includes an uninterruptible power supply (UPS); a network power switch (NPS) has been added by the site testing team. The NPS allows for manual or automatic power switching over a network connection. The $110 \mathrm{~V}$ circuit includes another UPS and two NPS systems (with eight switched power plugs per NPS); all of the computers and instrumentation are connected through these switches. In many cases, a computer that has crashed or a piece of equipment that is not responding can be recovered through a power cycle, so the NPS systems are critical for remote operations.

Each site has externally accessible web cameras that allow monitoring of the conditions on site. Images are taken during daylight hours at ten minute intervals; these images can be examined individually but are automatically composed into movies. These are used to check the safety of the site equipment, that the equipment is functioning properly, and to observe the weather conditions on site. The webcams are invaluable for determining the status of the site when communication is lost with a piece of equipment, or when the weather station is indicating suboptimal weather conditions.

\subsubsection{Network}

The networking system for each site starts with a connection to the outside world. These connections are either over a microwave antenna or through a direct fiber optic link, depending on the site. All sites have a sufficiently fast connection to allow continuous monitoring of the site and data backup (Sec. 4.2). The site testing local area network hardware includes a router and fast ethernet switches. The router is configured with a robust firewall, and uses custom ports for any external access to the site systems, to secure the site from any other type of access.

\subsubsection{Supervisor computer (SC)}

The center of the site testing system is the Supervisor Computer (SC). It controls the actions of the telescope, instruments, data management and monitors the status of all systems. The weather station is connected directly to a SC serial port, allowing the system to react quickly to weather conditions. The MASS is also connected to the $\mathrm{SC}$, as controlling the MASS through the robotic system is programmatically simpler with that connection. The SC operating system is a stripped down installation of RedHat Linux 9; at the time the system was developed, more modern versions of RedHat would not support the operation of the MASS, although that is no longer the case.

The robotic software control system is installed on the SC, and is divided into two separate parts: an extensive set of IDL ${ }^{12}$ scripts (with supporting Bash shell scripts and Expect scripts) that control operations of the Halfmann telescope, MASS and DIMM during night operations; and a second set of Bash scripts, C software, plotting software and other bits that control the operation of the other instruments, data management and status updates. The software controls the operation of the other instruments not connected to the SC as well, and manages the data flow and backup.

Time is an important factor in any astronomical observation, and the robotic system has to handle time appropriately since a consistent time record is essential for data quality to be assured. A built in ephemeris controls the timing of daily operations, correcting for changes in the rise and set times of the Sun. A network time protocol (NTP) server is installed and all site computers are synchronized to it; the SC is synchronized to the central server (Sec. 4.1) which is synchronized with standard internet NTP servers. This allows the site system to operate properly if the internet connection is disconnected for some reason. 
The SC is a full server system, with a web and mail server communicating information about the status of the system to the outside world; access to the system is limited to web and SSH connections for added security. The web site is a localized version of the one described in Sec. 4.1, which allows access to the data and displays the status of the system and the data as they are gathered.

\subsubsection{DIMM control computer}

The DIMM control computer (DC) controls the operations of the DIMM CCD through directions sent by the SC. The SC commands the DC to take a regular CCD image for pointing purposes, a dark frame or a DIMM data set; that command is transmitted to the DIMM CCD which then takes the frame. The DC uses a set of $\mathrm{C}++$ programs to communicate with the CCD and take the actual image; this software was developed in collaboration with Matt Longmire of SBIG to take pointing and data frames, and modified by TMT to take dark frames. An IDL script reads information from the SC to determine what image type to gather and how to reduce it. The DC then communicates the results back to the SC.

The DC operating system is Windows XP SP2; great care has been taken to develop a standard software installation that is stable. This computer is secured from internet connection except through a VNC program; this allows for direct communication with the Windows environment to solve issues that may occur.

The DIMM data analysis is performed using $\mathrm{C}++$ code developed by TMT. For pointing images, a centroid is calculated from the images to find the stellar position and telescope offsets necessary for accurate pointing and data acquisition. The data frames are of thirty second duration, where a portion of the CCD (40 pixels high, 100 wide) is read out once every 5 milliseconds. The portions are collapsed to a single line, and the seeing is measured from the motions of the centroids of the two stellar images in each line. This "one dimensional" reduction method was originally developed and implemented by the TMT site testing project.

\subsubsection{Instrument computer}

The Instrument Computer (IC) has the sonic anemometer and dust sensor attached directly to it through serial port connections. Each instrument uses software developed in house to gather data through the serial port. The dust sensor uses a Bash shell script to read the serial port output directly and then interprets the data into a convenient file format. The sonic anemometer raw data is read by a $\mathrm{C}++$ program written by Gordon Maclean of UCAR. Sonic anemometer data acquisition is continuous 24 hours a day; the instrument computer runs Bash shell scripts that monitor the incoming data stream and take appropriate corrective action if there is a problem. Both instruments can be controlled and stopped from the SC.

The IC uses a very stripped down version of RedHat Linux 9; the base system consists of the minimum installation plus development tools and an NTP daemon. This is done to keep the computer operating system as stable and efficient as possible.

\subsubsection{SODAR computer}

The SODAR control computer controls the operation of the XFAS and SFAS units. A commercial program from the SODAR manufacturer, Scintec, controls all operations of the SODAR, and gathers data on an automated twenty minute cycle. The SODAR operations are managed from the SC through a Bash script that determines when to start and stop SODAR functions. The SODAR software is Windows based, and the SODAR computer uses the same Windows XP SP2 installation as on the DC, but without the software used to control the DIMM CCD.

\subsubsection{Cube}

The Cube computer is an Intrinsyc CerfCube (model 250 or 405); it is essentially a "computer on a chip" type of system that consists of a very small circuit board with limited functionality. ${ }^{13}$ The Cube is intended as an overseer, and is used for two functions. First, it is an extra point of entry into the system through the network firewall (Sec. 3.2.3). Second, it is used to monitor the power systems of the computer cabinet. If there is a loss of power, the UPS sends a signal to the Cube that it is running on battery power only. At that point, the Cube and SC will work together to shut down all instrument and computer systems, leaving only the Cube and router running, as well as subsystems that include the webcams. This extends the amount of connection time with the 
site system as the battery demand is minimized. When power is restored, the computer systems are rebooted through the NPS units and all instruments are restarted when it is safe.

The Cube uses an imbedded version of Linux that is installed on the computers at the factory. The Cube has a Flash memory based disk system; extra space is added and new packages are installed to increase the functionality of the computer for TMT (the installed base system is very simple). Little additional software is necessary, as the Cube only monitors the power system and shuts down the equipment when required.

\subsubsection{Firewire disk storage}

The Stealths come standard with a small hard drive ( $\sim 30 \mathrm{~GB})$; this is not enough disk space to support operations for more than a few days. In order to have the system run continuously, or to run for several days independently without a network connection, additional storage is required. External disk storage using a Firewire disk was selected as an easy solution to the storage problem.

The DC would seem the obvious computer to connect an external disk to, as the DIMM CCD takes a large amount of data ( 1GB per night), and initially the system was configured that way. Windows did not work well in that configuration, so the Firewire drive was moved to the SC. This arrangement has the advantage that the Firewire drive is now shared among all the computers in the network. The SODAR and DC computers mount the Firewire drive from the SC through Samba, while the IC uses NFS; all write their data directly to the drive. The SC also archives the data each day to the Firewire drive, in preparation for the daily backup (Sec. 4.2).

There have been Firewire hard drive failures; as the drives operate continuously in inhospitable conditions, this is inevitable. The computer system is designed to recover from a Firewire drive failure and operations can continue, although closer monitoring and management of the disk space is required. The system will also stop operations if the Firewire drive (or backup system) becomes too full to accommodate more data.

\subsubsection{Instruments with computers: Halfmann telescope, IRMA and ASCA}

Each of these instruments comes equipped with a computer and operating system developed by the manufacturer. In each case, the site testing system works in conjunction with the instrument computer to achieve the desired operation of the instrument.

Halfmann telescope The Halfmann telescope system has many automatic control systems on it that, for example, close the dome on errors or power loss, or that move the motors continuously to set positions and hold them in place. These hardware systems are controlled through a SuSe Linux based operating system with manufacturer provided software. The telescope control computer can be commanded through an ethernet connection in a telnet session.

The TMT robotic software sends commands to the telescope system as required through the telnet session, and keeps constant track of the telescope status and responds appropriately to errors. The telescope system includes built in pointing model software for automated pointing and tracking of the target; the pointing model is built up manually by centering on stars and adding the star to a file. Once compiled from these data points, the model is very stable, and the telescopes only require a new model to be built after an optical realignment (following a mirror recoating or similar functions).

IRMA IRMA uses a "computer on chip" system (similar to the one in the Cube), with a Slackware Linux operating system. The University of Lethbridge team has supplied all of the software for the system; the TMT software consists only of scripts that will start and stop operations, park the instrument in a safe position when necessary and archive the data from IRMA on a daily basis.

ASCA As with IRMA, ASCA is a self contained unit; there is little interaction with the TMT system as ASCA is a simpler instrument. ASCA has a dedicated server, located in La Serena, Chile at the CTIO headquarters, which takes the images from the CCD and downloads them directly throughout the night. The images are downloaded to the dedicated server as they are taken on a five minute cycle; the images are processed by the dedicated server and displayed on a website. Since the telescope can be seen in the images, ASCA is also used as a check on the telescope status if there are problems during the night. 


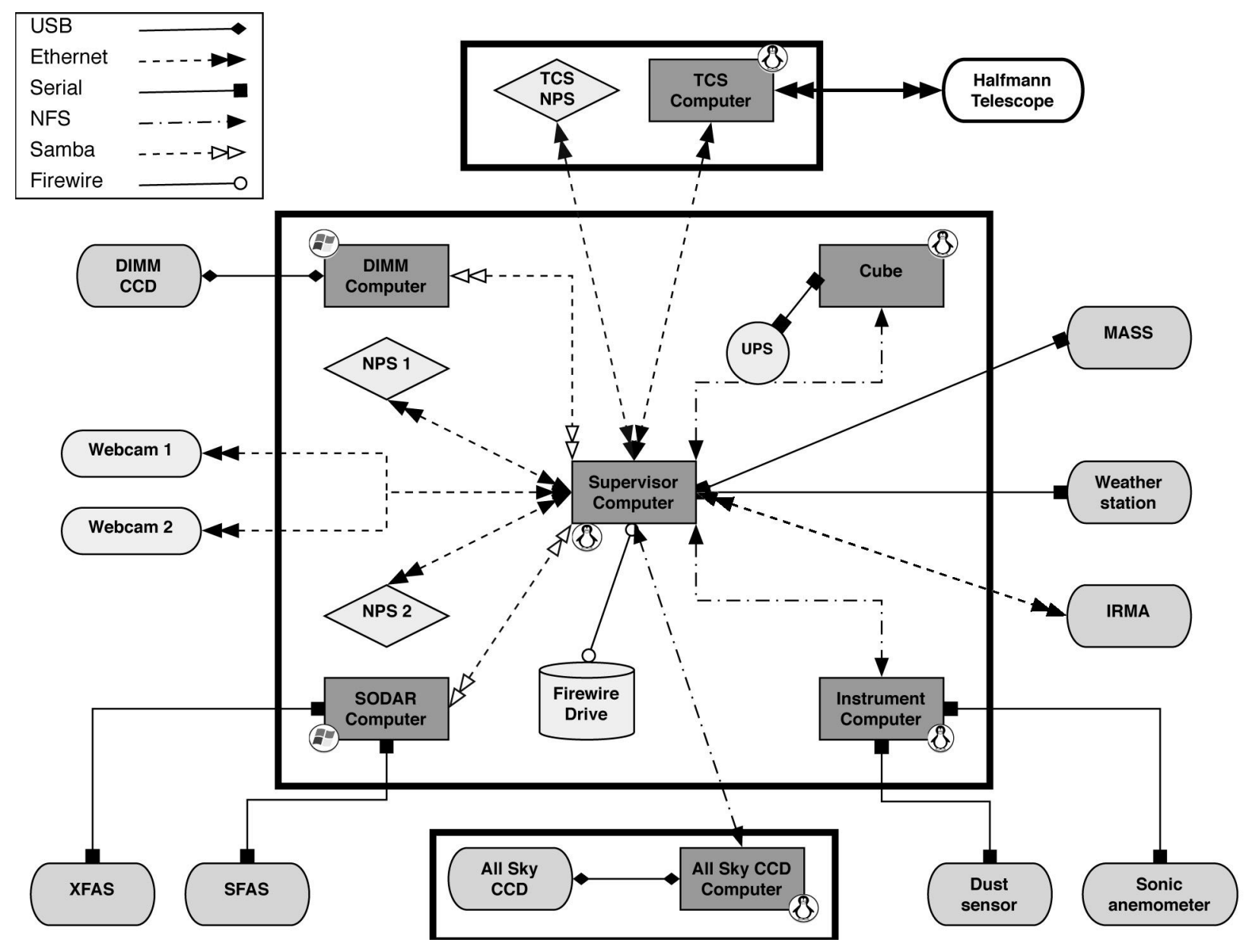

Figure 1. Schematic layout of the site testing computer systems. Connection types are coded using the symbols in the upper left corner, and computer operating systems are labeled. with icons. The external network connection to the router is not shown. Note that the Linux Penguin designates UNIX systems in general. Power connections are not shown for clarity.

\subsection{Putting It All Together}

A level beyond assembling the parts is making those computer and instrument subsystems work in an automated fashion, and in concert, night after night for two years or more. Putting the pieces together into a working instrument suite must be carefully thought out, as grafting in pieces latter requires more work than planning ahead of time.

Figure 1 is a schematic layout of the interconnections required for the TMT test sites. The large central box represents the computer cabinet installed on site; this is a weatherproof container that protects the computer equipment. Each site has the same physical cabinet layout, power and cabling setup, as uniformity in the setup leads to easier operations, maintenance and better reliability. The two other boxes are for the ASCA assembly (bottom) and Halfmann telescope electronics cabinet (top). The instruments are physically installed at various places unique to each testing site, so their placement in the figure is for diagrammatical purposes only.

\subsubsection{Installation}

A uniform operating system installation on the site testing computers is required in order to ensure that problems on a site are not due to incompatibilities in the computer systems, and that problems at different sites are not 
due to differences in the installations at each site. Great care was taken to develop a uniform installation for each of the systems, with the minimum of necessary software packages installed. ${ }^{14}$

There were several issues due to the nonuniform operating systems and extraneous packages before the standard, uniform installation was developed. After each site received upgraded computers with the new installation, operational efficiency increased noticeably. Therefore, any program should consider uniform computer installations, as much as possible, when deploying similar systems. The site testing software suite has been custom written by the site testing project team. The software will be improved and made more sophisticated throughout the lifetime of the site testing program, to increase the ability of the system to gather data and react to operational difficulties.

Nightly operations Each night, the operating system automatically launches the robotic system from a cron job. The robotic system waits for twilight, and then powers up the various subsystems through the NPS units, checks the weather and starts operations. The system automatically chooses a star (and switches targets through the night), points the telescope and centers on the target. The system then starts a cycle of MASS and DIMM observations, and weather and system checks. In the morning, the telescope dome closes, the telescope is parked and all instruments are shut down. The SODAR system is also started and stopped by the robotic system, gathering data in synchronization with the MASS/DIMM system, while ASCA has its own internal system to start and stop observations. The sonic anemometer, dust sensor, and IRMA run continuously.

If at any time the weather conditions go beyond a set of limits (in humidity, wind speed, temperature, dew point or rain/snow fall), the system will close the dome and park the telescope; if the weather returns to safe conditions the system will automatically restart operations. If the pointing images cannot find a target, the telescope will also stop for a fifteen minute weather pause; these are almost always due to clouds in the line of sight, but can also indicate a poor telescope pointing model. The weather system is robust enough to allow the robotic system to gather data right to the point when bad weather encroaches; this allows the testing project to

gather the maximum amount of data while protecting the system with a high level of reliability.

On most nights, the system runs without requiring human intervention or stopping due to technical problems. There is an error control system which is able to reset computers and instruments if there are glitches; it is upgraded as new errors are found, and on most nights is able to keep the system running. If an error occurs that the system cannot handle, it puts the telescope into a safe state and sends an email requesting assistance. There are additional fail safe systems in case the robotic software itself fails and is unable to stop the system. At that point a person can intervene and attempt to resuscitate the system.

Overall operations Supporting the gathering of data by the instrumentation is a suite of scripts that perform all of the management tasks for the system. One script controls when the other scripts run, and when nightly operations start and stop. Other scripts control the analysis of the data gathered from the previous night, create plots, and take the raw data and archive it. For TMT, archiving the data is critical as it is expected that a reanalysis of part or all of the data may be desirable at a later time. Scripts examine the system to determine which instruments are working and which are not, and gather the data from the weather station and webcams on a continuing basis. Each instrument can be started and stopped individually, and the entire site testing system can be shut down if necessary.

\section{CENTRAL SERVERS: COMPUTER SYSTEMS AND SOFTWARE}

Managing the separate sites would be extremely difficult without the centralized server systems in place as part of the testing program. The central servers keep the sites synchronized and archive the information necessary for data analysis.

\subsection{Web, Software Archive, Database and Mail Server}

This server is the heart of the site testing system, allowing the team to monitor the status of site operations in real time for all six sites, keep track of the software and configuration file changes, analyze data and communicate about the testing program. 


\subsubsection{Web services}

The site testing team has created several web services that are very useful in the execution of the site testing program. These include several features:

Status pages A suite of pages that monitor the site status on a real time (within a minute) basis. All aspects of the site operations are monitored, from telescope and computer equipment health to the operation of the instruments. Live data plots are generated as data are gathered. There are also pages for the webcams and ASCA cameras so the sites can be monitored visually day and night.

Site configuration A summary of the current configuration parameters at the sites, along with a verification of the instrument calibrations.

Observational data All of the observation data files, data plots, logs and error files are available for examination. A summary page shows the most recent data. A web form is used to send a daily update email for each site, which is a summary of the previous day of observation.

Problem logs A dynamic listing of problems with the site testing systems, with a library of solved problems for reference.

Database access Access to the database, discussed below.

Documentation A listing of manuals and other documentation for the site testing program.

Calendar An event calendar, to track travel and deadlines for the site testing team.

The web services are written in a mixture of Perl, PHP, Javascript, HTML and Bash shell scripts, with plots created by Bash shell scripts, Gnuplot scripts and Grace.

\subsubsection{Software archive}

With so many computers, it is essential to ensure that all the sites are running identical versions of the robotic software. The software is therefore constructed to use common scripts and program code throughout, with configuration files used to set variables inherent to each site. A Concurrent Version System (CVS) server is set up on the central server and all site software is synchronized to the CVS system. A check is made daily for changes in the software and configuration files. Additionally, all of the software from each site is synchronized to a copy on the central server; this backup allows the site to be recovered in case of computer failure, and has been used successfully several times.

\subsubsection{Database}

A PostgresSQL database was developed by A. J. Lavin ${ }^{15}$ to contain all of the data gathered during the site testing program. The database includes fields for the data values as well as flags for the quality of the data (valid, invalid, not checked yet, etc.), and is sorted by the date of observation and site. The database provides rapid access to any desired portion of the data, over a range of sites and times, in order to, for example, examine correlations between instruments. The database includes a web interface (for both data download and plotting online) and a command line interface, and is expandable to the limit of the available disk space.

\subsubsection{Mailing lists}

The mail server is a vital tool to communicate the status of all of the equipment used in the project and to plan operations, and is in constant use by the testing team. The mail server uses Mailman, an open source software project and standard mailing list package, to distribute daily update messages from the web server page as well as all regular communications.

The web/software/database/mail server is an Apple XServe G5, a dual 2.0 G5 processor system with 1GB of RAM, currently running OS X 10.4 Server. 
Table 2. The average disk space required for data gathered daily from each instrument at the testing sites. "Everything else" includes the rest of the instrumentation data files as well as logs, plots and other miscellaneous files.

\begin{tabular}{|l|c|c|}
\hline Instrument & Size, uncompressed (MB) & Size, compressed (MB) \\
\hline ASCA & 1,800 & 700 \\
\hline DIMM CCD & 850 & 350 \\
\hline Sonic Anemometer & 240 & 55 \\
\hline SODAR & 40 & 3 \\
\hline Everything else & 30 & 12 \\
\hline Total & 2,960 & 1,120 \\
\hline
\end{tabular}

\subsection{Data Archive Server}

An immense amount of data is gathered at each of the six testing sites every day. Table 2 is a summary of the major data contributors, in terms of file size; each day one site produces more than one gigabyte of compressed data, on average. That is 6.5 gigabytes of compressed data per day output by all the sites, or 2.3 terabytes per year. The vagaries of weather and instrument problems reduce this number somewhat, but not enough to make a large difference in the required disk space.

While the Firewire drive on site is large enough to hold several months of data (usually $~ 200 \mathrm{~GB}$ of space is available), it is operating in conditions that are not conducive to hard drive health. Several Firewire drives have failed in the course of this project, so even having two in place (and exchanging them for new ones as they fill) is not a reliable data archiving method. The site network connections are sufficiently fast to transfer the data within an hour or two, making transmission offsite a feasible alternative for archiving the data. To hold the amount of data from all of the sites, a large Redundant Array of Independent Disks (RAID) is used; this is the simplest way to create a large disk that contains all of the data in one place. The data are downloaded to this array, which is connected directly to the data archive server through a fiber optic connection.

Each day, upon the completion of the observing night, a script on the SC creates an archive of the data on the site computer network. Once that is completed, the script signals the data server that the archive is prepared through the use of a status file. The data archive server runs a periodic script that automatically logs into the SC and downloads the data archive created by the SC. The data server continues to attempt to download the data throughout the day until the archived data are downloaded or the next day's observations are prepared for archiving. Once the download is completed successfully, the status file is removed.

Two additional procedures are in place to secure the data archive. Once each week, a second script on the data archive server synchronizes the entire data set on the site with the server archive; this gathers up any files that were missed by the daily download. As this runs every week, it continually ensures that the data archive is complete. The data are also written to DVDs periodically (by a person instead of automatically); these hard copies are kept safe in case the data server is damaged.

The data server is an Apple XServe G5, a dual 2.3 G5 processor system with 1GB of RAM, currently running OS X 10.4 Server. The RAID is an Apple XServe RAID, with two RAID 5 arrays with 5.6TB of total disk space, which corresponds to two arrays of $2.18 \mathrm{~TB}$ of real disk space.

\subsection{Chilean Backup Server}

A third server and second RAID array has been deployed at the CTIO operation base in La Serena, Chile as an emergency backup system. Each day, the software, website and data from the web server and data server are synchronized to the Chilean server. The Chilean backup server does not communicate with the testing sites directly, but can be quickly configured to do the same automated backup and other tasks the other two servers do on a daily basis. 
If necessary, the backup server could take over all of the central server tasks; since this would be required only if the two servers in Pasadena were seriously compromised, the hope is that this will never be necessary. In the worst case scenario, it would require a short time (on the order of an hour) to switch operations to the backup server; no data would be lost from the sites in this process unless the site computers also failed at the same time.

The backup server is an Apple XServe G5, in this case a single 2.0 G5 processor system with 512MB of RAM, currently running OS X 10.4 Server. The RAID is an Apple XServe RAID, with one RAID 5 array of 2.8TB and a second of $1.75 \mathrm{~TB}$; these correspond to $2.18 \mathrm{~TB}$ and $1.37 \mathrm{~TB}$ of real disk space respectively.

\section{CONCLUSION}

The robotic system described in this paper is currently taking data on six sites, and will continue to do so until the completion of the site testing program in mid-2007. The final site selection for TMT depends on these systems gathering as much data as possible, making the operation of the robotic system a critical part of TMT. One of these systems is likely to continue monitoring site conditions after TMT is completed, a reminder of the program that put TMT onto one of the best sites for astronomical observations in the world.

\section{ACKNOWLEDGMENTS}

The authors gratefully acknowledge the support of the TMT partner institutions. They are the Association of Canadian Universities for Research in Astronomy (ACURA), the Association of Universities for Research in Astronomy (AURA), the California Institute of Technology and the University of California. This work was supported, as well, by the Canada Foundation for Innovation, the Gordon and Betty Moore Foundation, the National Optical Astronomy Observatory, which is operated by AURA under cooperative agreement with the National Science Foundation, the Ontario Ministry of Research and Innovation, and the National Research Council of Canada."

\section{REFERENCES}

1. V. Kornilov, A. Tokovinin, O. Vozyakova, A. Zaitsev, N. Shatsky, S. Potanin, and M. Sarazin, "MASS: a monitor of the vertical turbulence distribution," Proc. SPIE 4839, pp. 837-845, 2003.

2. L. Wang, M. Schöck, G. Chanan, W. Skidmore, E. Bustos, J. Seguel, and R. Blum, "High Accuracy DIMM Measurements for the TMT Site Testing Program," in Proc. SPIE, Astronomical Telescopes and Instrumentation 2006 6267-40, this proceeding, May 2006.

3. Santa Barbara Instrument Group Website, http://www.sbig.com.

4. W. Skidmore, T. Travouillon, and R. Riddle, "Evaluation of sonic anemometers as highly sensitive optical turbulence measuring devices for the Thirty Meter Telescope site testing campaign," in Proc. SPIE, Astronomical Telescopes and Instrumentation 2006 6267-48, this proceeding, May 2006.

5. Met One Instruments, Inc. Website, http://www.metone.com/.

6. T. Travouillon, "SODAR Calibration for Turbulence Profiling in TMT Site Testing," in Proc. SPIE, Astronomical Telescopes and Instrumentation 2006 6267-50, this proceeding, May 2006.

7. D. E. Walker, H. E. Schwarz, and E. Bustos, "Monitoring the night sky with the Cerro Tololo All-Sky camera for the TMT and LSST projects," in Proc. SPIE, Astronomical Telescopes and Instrumentation 2006 6267-51, this proceeding, May 2006.

8. Monitor Sensors (Aust) Pty Ltd. Website, http://www.monitorsensors.com/.

9. R. R. Phillips and D. A. Naylor, "Initial operations of an water vapour monitor (IRMA) at Gemini South, Las Campanas Observatories and in the TMT site testing role," in Proc. SPIE, Astronomical Telescopes and Instrumentation 2006 6269-204, this proceeding, May 2006.

10. Advanced Energy Group Website, http://www.solar4power.com.

11. Stealth Computer Corporation Website, http://www.stealthcomputer.com/home.htm.

12. Research Systems, Inc. Website, http://www.rsinc.com/.

13. Intrinsyc Software International Website, http://www.intrinsyc.com/.

14. R. L. Riddle, "TMT Site Testing Computer Systems," Manual, Thirty Meter Telescope, 2006.

15. A. J. Lavin Website, http://www.picturepusher.com/. 